\title{
Re: Using Space-Based Investigations to Inform Cancer Research on Earth
}

\author{
J.L. Becker, G.R. Souza
}

Nano3D Biosciences, Houston, TX

Nat Rev Cancer 2013;13:315-327.

\section{EDITORIAL COMMENT}

In the Universe, four main forces are effective. These are weak nuclear force, strong nuclear force, electromagnetic force and gravitational force. Gravity is the attractive force between all matter. In space, the force of gravity is diminished, resulting in microgravity. Currently, there are various studies about microgravity and its effect on biological functions in the literature. In this review, the authors reported effects of microgravity on cancer culture studies. In these studies, some devices are used such as rotating wall vessel (RWV) bioreactor, clinostat, random positioning machine (RPM) (3D clinostat), and magnetic levitation. These 3D cell culture studies are focused on oncology. We can find some studies that especially focused on culture or co-culture of prostate cancer in the microgravity conditions. These authors suggested that effects of microgravity on the oncological cultures in the space station $\left(10^{-4}\right.$ and $\left.10^{-6} \mathrm{~g}\right)$ were immune cell changes, alterations in gene expression, effects on cell signaling, effects on apoptosis, cytoskeletal changes, and alterations in cell shape. Microgravity environment of space includes lack of sedimentation, reduced fluid shear, cellular co-location and 3D multicellular growth. Microencapsulation technology was developed in like these conditions using a xenograft model of prostate cancer. Space presents an unlimited horizon for discovery. Microgravity can further our understanding of the fundamental role of gravity in cancer cell growth and function. These studies will expand our knowledge necessary for improving treatment options and exploring the etiopathogenesis of cancer.

Fehmi Narter, MD, PhD

Basic Science doi: $10.4274 / j u s .2015 .02 .010$

\section{Re: Modeling Tissue Morphogenesis and Cancer in 3D}

\section{Kenneth M. Yamada1, Edna Cukierman2}

1 Laboratory of Cell and Developmental Biology, National Institute of Dental and Craniofacial Research, National Institutes of Health, Maryland, United States of America

2Fox Chase Cancer Center, Tumor Cell Biology, Basic Science Division, Philadelphia, United States of America

Cell 2007;130;601-610.

\section{EDITORIAL COMMENT}

What does determine the shapes of the organs? Why the shape of the heart is like a drop? Or how kidneys are shaped like beans? Tissues and organs are three dimensional (3D). However, their formation, function and pathology have often depended on two-dimensional (2D) cell culture studies. In context of organogenesis, morphogenesis determines the shape of organs and these processes are named as mophogenesis. Morphogenesis includes cell-cell adhesion, extracellular matrix and cell contractility. For example, retinoic acid may also act as morphogenesis. Currently, there are many studies about morphogenesis in the literature. In one of them, the authors have reported that studies with 3D model systems have repeatedly identified complex interacting roles of matrix stiffness and composition, integrins, growth factor receptors and signaling in development and cancer. Differentiation, motility, cell shape, gene expression, growth, and morphogenesis researches are more effective in $3 \mathrm{D}$ cell cultures than in $2 \mathrm{D}$ cell cultures. For most studies, the tissues must be thin enough to permit adequate oxygenation and nutrition of the tissue interior, e.g. less than $0.3 \mathrm{~mm}$ thick. Especially, tumor proliferation, progression, invasion and metastasis abilities should be studied in 3D cell culture. These insights suggest that plasticity, regulation and suppression of these processes can provide strategies and therapeutic targets for future cancer therapy. In the near future, morphogenesis studies will be more determinant for tissue engineering, replacement therapies and cancer etiopathogenesis research.

Fehmi Narter, MD, PhD 